\title{
Abelhas e vespas solitárias em ninhos-armadilha na Reserva Biológica Guaribas (Mamanguape, Paraíba, Brasil)
}

\author{
Antonio José Camillo Aguiar ${ }^{1,2}$ \\ Celso Feitosa Martins ${ }^{1}$
}

\begin{abstract}
Trap-nesting solitary bees and wasps (Hymenoptera) in Guaribas Biological Reserve (Mamanguape, Paraíba, Brasil). The trap-nesting bees of Guaribas Biological Reserve were studied during one year. Three areas with different vegetation types were sampled, open savanas, closed forest, and a mosaic of these two types. Twelve species of trap-nesting bees were observed, four of which are parasites. The most abundant species were Centris tarsata (Smith, 1879), C. analis (Fabricius, 1804), Tetrapedia diversipes Klug, 1810, and Mesocheira bicolor (Fabricius, 1804). Nesting of Centris tarsata and Mesocheira bicolor occurred mainly during the dry season, while Centris analis and Tetrapedia diversipes did not show any seasonal preferences. Mesocheira bicolor occurred only in the nests of $C$. tarsata, parasitizing more than $50 \%$ of the nests and representing $23.2 \%$ of the individuals emerged in the host nests. The mosaic area yielded a greater abundance and diversity. Comparing the sampling methods of trap-nests and entomological nets, conducted simultaneously in the same area, in relation to diversity of trap nesting bees, it was observed a great similarity in terms of composition and differences of abundance in species sampled. Some hypothesis are discussed to explain a reason for the greater diversity and abundance of the mosaic area in relation to the other areas.

KEY WORDS. Apoidea, Apiformes, bees, Tabuleiro, trap-nests
\end{abstract}

As abelhas pertencem aos Apoidea Apiformes, estimadas em 30.000 espécies, com uma grande variedade de hábitos de nidificação, desde espécies solitárias com poucas células de cria, até espécies sociais com milhares de células (RoUBIK 1989; MICHENER 2000). As cavidades preexistentes em madeira, utilizadas para nidificação de algumas espécies de abelhas e vespas, são constituídas por orifícios feitos por larvas de outros insetos e também por galhos ocos. Os orifícios adequados para cada espécie de abelha ou vespa nidificante em cavidades preexistentes representam um recurso escasso no ambiente que limitam o crescimento das populações destas espécies (VINSON et al. 1993).

A metodologia de amostragem com ninhos-armadilhas permite a obtenção de informações sobre a diversidade e abundância de espécies nidificantes em cavidades preexistentes, assim como sobre a biologia das espécies, materiais de construção utilizados, arquitetura dos ninhos, recursos fornecidos para as larvas e biologia das espécies parasitas (GARÓFALO 2000). Alguns estudos têm inferido a possibilidade de se utilizar as espécies nidificantes em ninhos-armadilhas como

1) Departamento de Sistemática e Ecologia, Universidade Federal da Paraíba. 58000-900 João Pessoa, Paraíba, Brasil.

2) Bolsista CAPES.

Revta bras. Zool. 19 (Supl. 1): 101 - 116, 2002 
bioindicadores de qualidade do ambiente e diversidade em programas de conservação ambiental (BEYER et al. 1987; FRANKIE et al. 1998; TSCHARNTKE et al. 1998; MORATO \& CAMPOS 2000), e também a sua utilização como espécies manejáveis para incremento da polinização de espécies agrícolas (WILLIAMS 1996).

A amostragem com ninhos-armadilha além de ser um método relativamente simples de levantar a diversidade de espécies nidificantes em cavidades preexistentes, amostra somente as espécies que nidificam na área evitando as espécies que estejam apenas transitando no local de estudo (CAMILLO et al. 1995, TSCHARNTKE et al. 1998).

A metodologia dos ninhos-armadilha também permite réplicas (espaciais e temporais) padronizadas da amostragem, através da exposição de igual número e tipos de ninhos-armadilha, evitando diferenças provenientes de diferentes esforços amostrais realizados por diferentes coletores utilizando rede entomológica (TSCHARNTKE et al. 1998).

Um marco inicial para os estudos com ninhos-armadilhas foi o estudo realizado por KROMBEIN (1967) na América do Norte. Alguns trabalhos com ninhos-armadilha foram realizados no Brasil, em áreas de cerrado (SERRANO 1978; GARÓFALO et al. 1989, 1993; CAMILlO et al. 1995; PEREIRA et al. 1999), em dunas litorâneas (VIANA et al. 2001) e na floresta amazônica (MORATO et al. 1999; MORATO \& CAMPOS 2000); na Costa Rica em florestas sazonais secas (FRANKIE et al. 1988, 1993), na Alemanha, em áreas de cultivo agrícola (TSCHARNTKE et al. 1998).

Os objetivos do presente trabalho foram: 1) levantar a riqueza e a diversidade de espécies de abelhas nidificantes em cavidades preexistentes em madeira na Reserva Biológica Guaribas; 2) investigar a existência de padrões sazonais de nidificação das espécies de abelhas; 3 ) comparar as áreas da reserva com diferentes formações vegetais quanto à riqueza e a diversidade das abelhas nidificantes nos ninhos-armadilha e; 4) comparar a eficiência dos ninhos-armadilha em relação à coleta com rede entomológica para a diversidade de abelhas nidificantes em cavidades preexistentes.

\section{MATERIAL E MÉTODOS}

\section{Área de Estudo}

A Reserva Biológica Guaribas (Rebio Guaribas) está localizada no extremo Nordeste do Estado da Paraíba. O clima local, segundo Köppen, é do tipo As, quente e úmido, com estação seca no verão (novembro a março) e chuvosa no outono-inverno (abril a outubro). A pluviosidade está entre 1750 e $2000 \mathrm{~mm}$ anuais, com temperaturas médias anuais em torno de $24-26^{\circ} \mathrm{C}$ (NIMER 1979). A Rebio Guaribas apresenta duas áreas separadas, uma no município de Mamanguape e a outra no município de Rio Tinto. Este estudo foi realizado na parcela localizada no município de Mamanguape (6 $6^{\circ} 40^{\prime}-6^{\circ} 45^{\prime}$ 'S, e $\left.35^{\circ} 07^{\prime}-35^{\circ} 12^{\prime} \mathrm{W}\right)$.

A Rebio Guaribas está incluída na área Mamanguape/Baía Formosa (Paraíba/Rio Grande do Norte), definida como de extrema importância biológica para a conservação e manejo da Mata Atlântica (MMA 2000). A parcela da Rebio Guaribas estudada apresenta 3994,2 hectares com duas formações vegetais bastante distintas, 
conforme a classificação em regiões fito-ecológicas (SALGADO et al. 1981): Floresta Estacional Semi-decidual (Mata Atlântica) de formação secundária e Savana Arbórea Aberta (Tabuleiro).

No nordeste brasileiro, a denominação Tabuleiro é utilizada tanto para o conjunto formado pelos baixos planaltos próximos ao litoral com solo arenoso lixiviado, como para a sua vegetação savânica semelhante ao Cerrado. Os Tabuleiros ocorrem nas áreas continentais adjacentes à Restinga, estendendo-se sobre as planícies arenosas formadas sobre o Grupo Geológico Barreiras (OLIVEIRA-FILHO \& CARVAlHo 1993).

Os ninhos-armadilha foram instalados em três áreas: área I composta por vegetação savânica típica de tabuleiros abertos formada em sua maior parte por gramíneas e espécies herbáceas com aglomerações esparsas de vegetação arbórea arbustiva; área II com uma vegetação em mosaico representando uma área de transição ecológica entre as áreas I e III, com espécies vegetais comuns às duas áreas, e formações abertas margeadas por Mata Atlântica secundária; e a área III composta por Mata Atlântica secundária.

\section{ninhos-armadilha}

Os ninhos-armadilha foram elaborados com tubos de cartolina preta inseridos em orifícios feitos em blocos de madeira, semelhante ao método utilizado por CAMILLO et al. (1995).

Foram instaladas três unidades amostrais em cada área. Cada unidade amostral era constituída por 93 ninhos-armadilha, sendo 21 orifícios de $0,4 \mathrm{~cm}$ de diâmetro, 21 de $0,6 \mathrm{~cm}, 21$ de $0,8 \mathrm{~cm}, 10$ de $1,1 \mathrm{~cm}, 10$ de $1,3 \mathrm{~cm}$, e 10 de $1,5 \mathrm{~cm}$, com aproximadamente $11 \mathrm{~cm}$ de comprimento, exceto o de diâmetro 0,4 que possuía $8 \mathrm{~cm}$ de comprimento. No total, foram instalados 837 ninhos-armadilha.

Os ninhos-armadilha foram inspecionados mensalmente, no período de abril de 1999 a abril de 2000. Os ninhos ocupados, que não apresentavam atividade de construção, foram retirados e substituídos por tubos de cartolina vazios. Durante todo o experimento, sempre havia tubos vazios disponíveis para nidificação. Os tubos ocupados foram levados para o laboratório, sendo cada um inserido em uma garrafa plástica, com pequenas perfurações para permitir a aeração, e colocados em local seco e arejado. Após a emergência de todos os imagos dos ninhos, estes foram sacrificados com acetato de etila e os ninhos foram abertos para a exame das estruturas internas.

\section{Amostragem com rede entomológica}

Foram realizadas 403 horas de coleta ao longo de 12 meses e 51 dias (abril/1999 a março/2000), média de 33,5 horas mensais. A metodologia empregada foi modificada com base no método padrão estabelecido por SAKAGAMI et al. (1967).

Foi estabelecida uma linha de transecção de aproximadamente 1500 metros de comprimento na área II, percorrendo-se as áreas abertas próximo à borda das áreas mais fechadas, buscando amostrar as áreas de contato entre a Floresta Estacional e a Savana Arbórea Aberta. A linha de transecção foi percorrida men- 
salmente, durante três a quatro dias consecutivos, entre as 5:00 e 17:00 horas, observando-se as floradas até uma distância aproximada de $15 \mathrm{~m}$ de cada lado da trilha, abrangendo uma área de aproximadamente 4,5 hectares.

Os espécimes coletados nos ninhos-armadilha e com rede entomológica encontram-se depositados na coleção de referência do Laboratório de Entomologia, Departamento de Sistemática e Ecologia, Universidade Federal da Paraíba, João Pessoa.

\section{Análise dos dados}

Para relacionar a abundância de ninhos e indivíduos emergidos com a precipitação pluviométrica foram calculados índices de correlação de Spearman $\left(\mathrm{r}_{s}\right)$ utilizando o programa SPSS versão 9.0 para Windows.

Os dados de precipitação pluviométrica foram fornecidos pela Estação Mamanguape ( $\left.6^{\circ} 39^{\prime} \mathrm{S}, 35^{\circ} 07^{\prime} \mathrm{W}\right)$, pertencente ao Laboratório de Meteorologia, Recursos Hídricos e Sensoriamento Remoto da Paraíba (LMRS, Paraíba).

A diversidade de cada área foi estimada através do índice de Shannon-Wiener (H'). Os índices foram comparados através do teste t de HUTCHESON (1970), utilizando-se o programa Bio-Dap (MAGURRAN 1988). Com o propósito de testar se a razão sexual das espécies difere do esperado (1:1) foi utilizado o método de $X^{2}$ (LUDWIG \& REYNOLDS 1988).

\section{RESULTADOS}

\section{Composição de espécies}

No período de abril de 1999 a abril de 2000 , foram coletados 176 ninhos, sendo $138(78,4 \%)$ de abelhas e 38 de vespas, destes $31(17,6 \%)$ foram de Sphecidae e $7(4 \%)$ de Vespidae. Quanto ao número de ninhos por família de abelhas (ROIG-ALSINA \& MiCHENER 1993), dos 138 ninhos de abelhas, 93,4\% foram ocupados por Apidae e 6,5\% por Megachilidae.

Foram amostradas cinco espécies de Apidae, sendo quatro nidificantes e uma espécie parasita. Para Megachilidae foram encontradas sete espécies, sendo quatro espécies nidificantes e três parasitas. As espécies de abelhas nidificantes nos ninhos-armadilha foram: Centris (Heterocentris) tarsata (Smith, 1874), Centris (Heterocentris) analis (Fabricius, 1804), Tetrapedia diversipes Klug, 1810, Euglossa (Euglossa) cordata (Linnaeus, 1758), Epanthidium tigrinum Schrottky, 1905, Megachile (Neomegachile) brethesi Schrottky, 1909, Megachile (Sayapis) dentipes Vachal, 1909 e Megachile (Pseudocentron) sp. As espécies de abelhas parasitas foram Mesocheira bicolor (Fabricius, 1804) nos ninhos de Centris tarsata; Coelioxys (Neocoelioxys) sp. e Coelioxys sp. 2 nos ninhos de Centris analis e Centris tarsata; e Coelioxys sp. 1 nos ninhos de Megachile (Pseudocentron) sp. (Tab. I). As espécies Centris tarsata, Centris analis e Tetrapedia diversipes apresentaram o maior índice de nidificação (Fig 1, Tab. I).

Nos 176 ninhos coletados, emergiram 619 indivíduos, incluindo os parasitas. Do número total de indivíduos, as abelhas foram representadas por 79,6\%, sendo $73,5 \%$ Apidae e 6,1\% Megachilidae. Os Sphecidae representaram 14,5\%, Vespidae $4,6 \%$, Chrysididae $0,3 \%$, Coleoptera $0,3 \%$, Diptera $0,3 \%$ e Lepidoptera $0,1 \%$. 


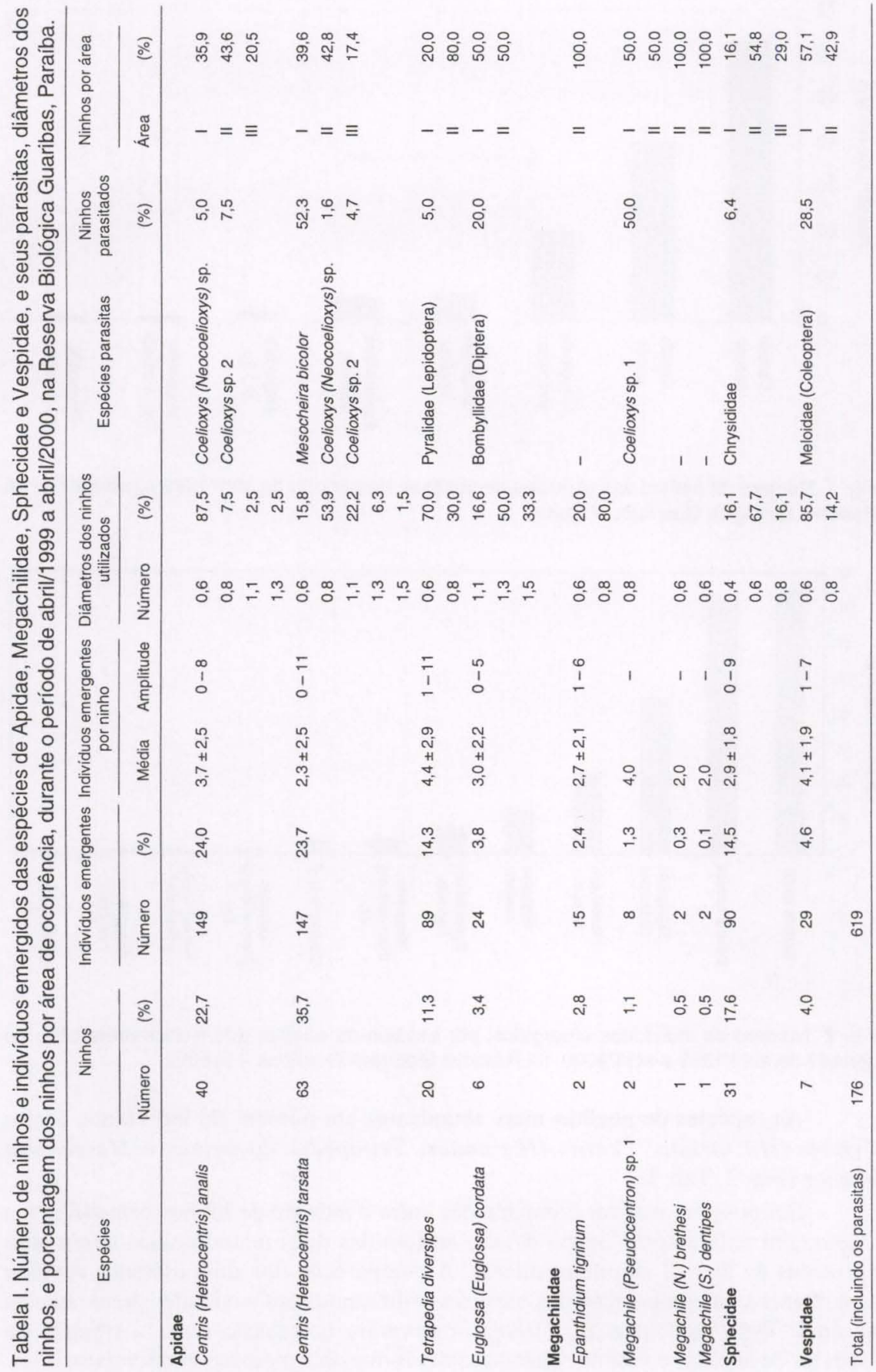

Revta bras. Zool. 19 (Supl. 1): 101 - 116, 2002 


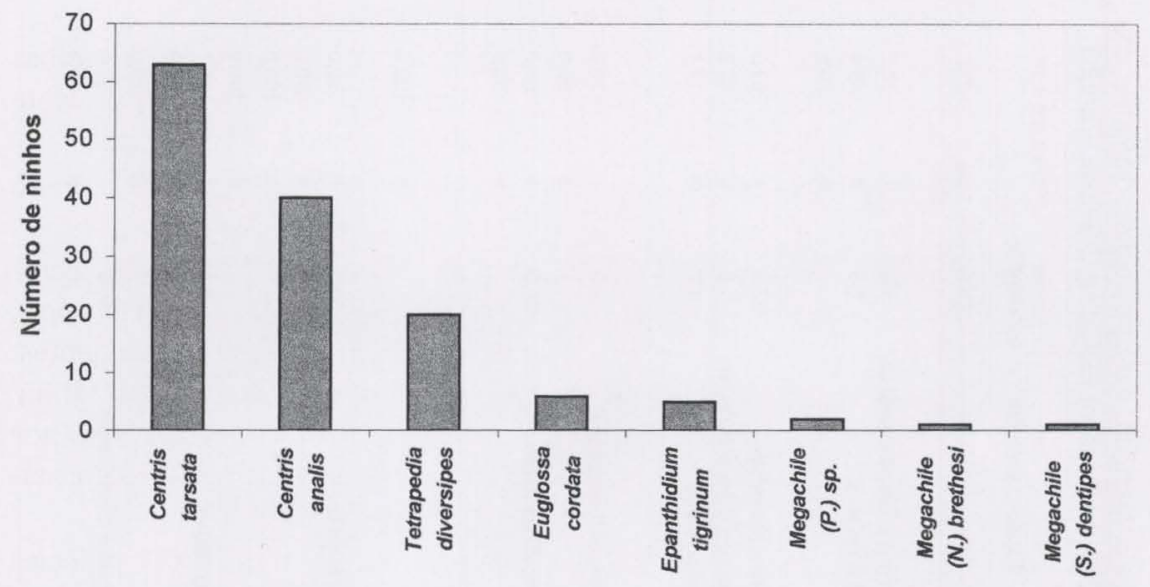

Fig. 1. Número de ninhos das espécies de abelhas, no período de abril/1999 a julho/2000, na Reserva Biológica Guaribas, Paraíba.

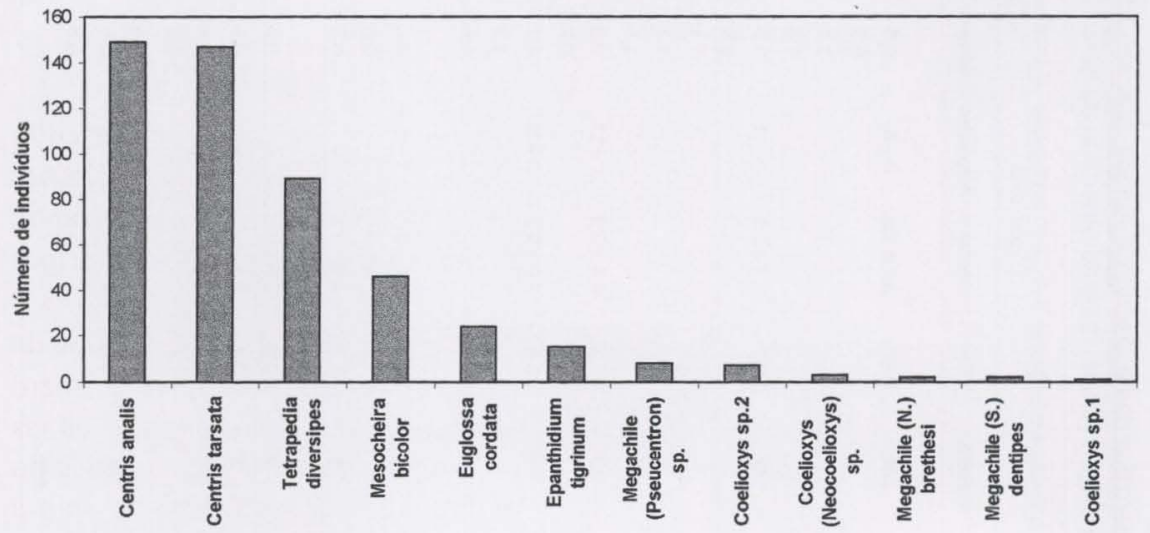

Fig. 2. Número de indivíduos emergidos, por espécie de abelha, dos ninhos-armadilha, no período de abril/1999 a abril/2000, na Reserva Biológica Guaribas, Paraíba.

As espécies de abelhas mais abundantes em número de indivíduos foram Centris (H.) tarsata, Centris (H.) analis, Tetrapedia diversipes e Mesocheira bicolor (Fig. 2, Tab. I).

Foi possível realizar comparações entre o método de ninhos-armadilha e a coleta com rede entomológica, devido ao fato das duas metodologias terem sido utilizadas na área II simultaneamente. A comparação dos dois métodos revelou semelhanças na composição das espécies nidificantes em cavidades preexistentes na área. TSCHARNTKE et al. (1998) observaram correlação entre a riqueza de espécies de abelhas e vespas coletadas através dos dois métodos em diversas áreas. 
Através do método com rede entomológica, foram coletadas 3022 abelhas de 115 espécies de abelhas, sendo destas 112 indivíduos (3,8\% do total) de vinte espécies $(17,4 \%)$ de abelhas nidificantes em cavidades preexistentes.

A espécie Mesocheira bicolor não foi amostrada sobre as flores, sendo abundante nos ninhos-armadilha. Centris (Xanthemisia) lutea Friese, 1899, C. (X.) ferruginea (Lepeletier, 1841), Coelioxys (Acrocoelioxys) praetextata Haliday, 1837, e espécies não identificadas de Megachile (3 spp.), Coelioxys (3 spp.), e Hylaeus Fabricius, 1793 ( $3 \mathrm{spp}$.), que nidificam em cavidades preexistentes, foram coletadas sobre as flores mas não foram encontradas nos ninhos-armadilha. FRANKIE et al. (1988), também não encontraram Centris (Xanthemisia) lutea nos ninhos-armadill , e inferem que esta espécie não é atraída para os ninhos-armadilhas, apesar de nidificarem em cavidades preexistentes.

Por outro lado, foram observadas diferenças na abundância das espécies coletadas pelos dois métodos. As seguintes espécies coletadas nos ninhos-armadilha: Centris analis, Tetrapedia diversipes, Euglossa cordata, Epanthidium tigrinum, Coelioxys (Neocoelioxys) sp., Coelioxys sp.1 e Coelioxys sp.2, sendo as duas primeiras bem abundantes, apresentaram um baixo número de indivíduos amostrados utilizando rede entomológica.

\section{Sazonalidade}

Não foi observada uma variação no número de nidificações das vespas entre as estações seca e chuvosa. No caso das abelhas, o maior número de nidificações (35 ninhos, 25\%) ocorreu em novembro, durante o período seco (Fig. 4). Entretanto, não foram observadas correlações significativas entre o número de ninhos de vespas ou de abelhas e a precipitação pluviométrica.

Com o propósito de verificar a relação entre a abundância de ninhos ou de indivíduos e a precipitação pluviométrica, foram analisados os padrões de sazonalidade das espécies mais abundantes. Foram consideradas predominantes, as espécies de abelhas que apresentaram uma proporção maior de nidificações do que o total de ninhos coletados dividido pelo número de espécies, ou seja a proporção esperada caso não houvesse dominância. O mesmo critério foi utilizado para a abundância em número de indivíduos. Os dados referentes à distribuição da abundância das espécies de abelhas predominantes ao longo de um ano, em número de ninhos e indivíduos emergidos estão representados, respectivamente, nas figuras 3 e 4 .

Pode-se notar que Centris tarsata apresentou maior índice de nidificação no período seco, enquanto Centris analis foi mais comum no fim da estação seca e início da estação chuvosa (novembro, janeiro e fevereiro) (Fig. 3). Porém, somente C. tarsata apresentou correlação negativa significativa entre o número de nidificações e a precipitação pluviométrica $\left(\mathbf{r}_{s}=-0,578 ; \mathrm{p}<0,05\right)$.

Quanto ao número de indivíduos, os padrões apresentados foram semelhantes aos observados quanto ao número de ninhos, porém, não foram observadas correlações significativas com a precipitação pluviométrica (Fig. 4). 

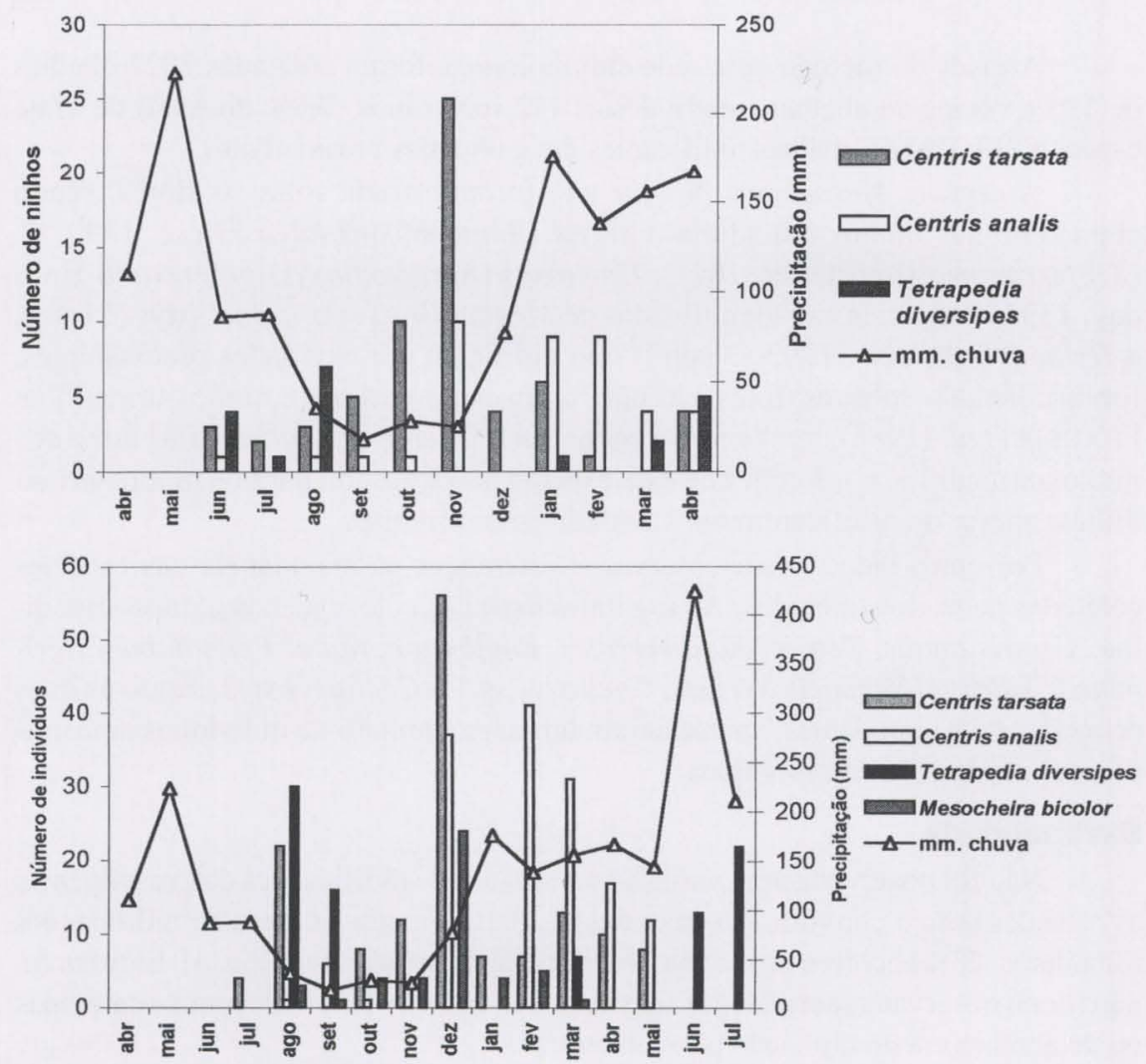

Figs 3-4. Número de ninhos (3) e de indivíduos emergidos (4) das espécies de abelhas mais abundantes e precipitação pluviométrica, dos ninhos coletados durante o período de abril/1999 a abril/2000, na Reserva Biológica Guaribas, Paraíba.

\section{Diâmetro dos Ninhos}

As abelhas utilizaram praticamente todos os diâmetros de ninhos-armadilha oferecidos, com exceção de $0,4 \mathrm{~cm}$. Os ninhos com maior porcentagem de nidificação foram aqueles com diâmetro de $0,6 \mathrm{~cm}(47,1 \%)$ e $0,8 \mathrm{~cm}(33,3 \%)$. As vespas ocuparam ninhos-armadilha de somente três diâmetros; os Sphecidae utilizaram em $67,7 \%$ dos casos diâmetros de $0,6 \mathrm{~cm}, 16,1 \%$ em $0,4 \mathrm{~cm}$, e $16,1 \%$ em $0,8 \mathrm{~cm}$; os Vespidae ocuparam em $85,7 \%$ dos casos ninhos com $0,6 \mathrm{~cm}$ de diâmetro e $14,2 \%$ em $0,8 \mathrm{~cm}$ (Tab. I).

Com relação à preferência das espécies de abelhas por diferentes diâmetros, $87,5 \%$ dos ninhos de $C$. analis ocorreram em orifícios com $0,6 \mathrm{~cm}$ de diâmetro; $C$. tarsata nidificou em 53,9\% dos casos em diâmetros de $0,8 \mathrm{~cm}$, e Tetrapedia diversipes apresentou $70 \%$ dos ninhos em diâmetros de $0,6 \mathrm{~cm}$. Os diâmetros de $1,1 \mathrm{~cm}, 1,3 \mathrm{~cm}$ e $1,5 \mathrm{~cm}$ foram utilizados preferencialmente por Euglossa cordata e em menor número por C. tarsata e C. analis (Tab. I). 


\section{Ocorrência das espécies nas diferentes áreas}

A percentagem do número de ninhos ocupados nas três áreas da Rebio Guaribas está representada na tabela I. Mais de 50\% dos ninhos de abelhas e vespas ocorreram na área II, e a área III foi a que apresentou o menor número de ninhos de abelhas (Fig. 5). Além do maior número de ninhos, a área II também apresentou um maior número de espécies.

A área II apresentou um índice de diversidade significativamente maior que as demais áreas, embora a área III tenha apresentado uma maior equitatividade (Tab. II).

Tabela II. Índice de diversidade $\left(H^{\prime}\right)$ e equitatividade $(E)$ das três áreas amostradas com ninhos-armadilha, no período de abril/1999 a abril/2000, na Reserva Biológica Guaribas, Paraíba.

\begin{tabular}{lccc}
\hline Índices & Área I & Área II & Área III \\
\hline$H^{\prime}$ & 1,08 & 1,52 & 0,69 \\
$E$ & 0,67 & 0,73 & 1,00 \\
\hline IX II & $\mathrm{t}=2,924 ; g \mid=95,4 ; p<0,01$ & & \\
IX III & $\mathrm{t}=3,379 ; g \mid=40,4 ; p<0,01$ & & \\
IIX III & $\mathrm{t}=9,646 ; g \mid=59,44 ; p<0,001$ & & \\
\hline
\end{tabular}

(t) Teste t de Hutcheson ( $\mathrm{gl}$ ) graus de liberdade.

Das 12 espécies de abelhas coletadas na área II, seis espécies também ocorreram na área I (Centris tarsata, C. analis, Tetrapedia diversipes, Euglossa cordata, Mesocheira bicolor, Megachile (Pseudocentron) sp.) e quatro na área III (C. tarsata, C. analis, M. bicolor e Coelioxys (Neocoelioxys) sp. Cinco espécies de abelhas (Epanthidium tigrinum, Megachile (S.) dentipes., M.(N.) brethesi, Coelioxys sp.1 e Coelioxys sp.2) foram encontradas somente na área II (Tab. I). Entre as três espécies de abelhas predominantes em número de nidificações, somente duas estiveram presentes nas três áreas: $C$. tarsata e $C$. analis (Tab. I, Fig. 6). Apesar do maior número de nidificações de $C$. tarsata na área II, na área I o número de indivíduos emergidos foi cerca de 50\% maior (Tab. I, Fig. 7).

\section{Parasitas}

Cerca de $28 \%$ dos ninhos de abelhas estavam parasitados, sendo que o número de indivíduos emergidos de espécies parasitas representou $12,5 \%$ do total. Os ninhos de Vespidae foram parasitados por uma espécie não identificada de Meloidae (Coleoptera), e os Sphecidae, por uma espécie não identificada de Chrysididae (Hymenoptera) (Tab. I).

Aproximadamente $51 \%$ dos ninhos de Centris tarsata estavam parasitados. Do total de 198 indivíduos emergidos dos ninhos de C. tarsata, 25,7\% eram parasitas, sendo 23,2\% de Mesocheira bicolor, 2,0\% Coelioxys sp. 2 e 0,5\% Coelioxys (Neocoelioxys) sp. Durante a estação seca, houve um aumento proporcional no número de nidificações de $C$. tarsata e de Mesocheira bicolor, sendo que cerca de $60 \%$ dos ninhos de C. tarsata foram parasitados por $M$. bicolor nesta estação. Foi observada correlação significativa entre o número de ninhos de $C$. tarsata por mês e o número de ninhos parasitados por Mesocheira bicolor $\left(\mathrm{r}_{s}=0,781 ; \mathrm{p}<0,01\right)$. 

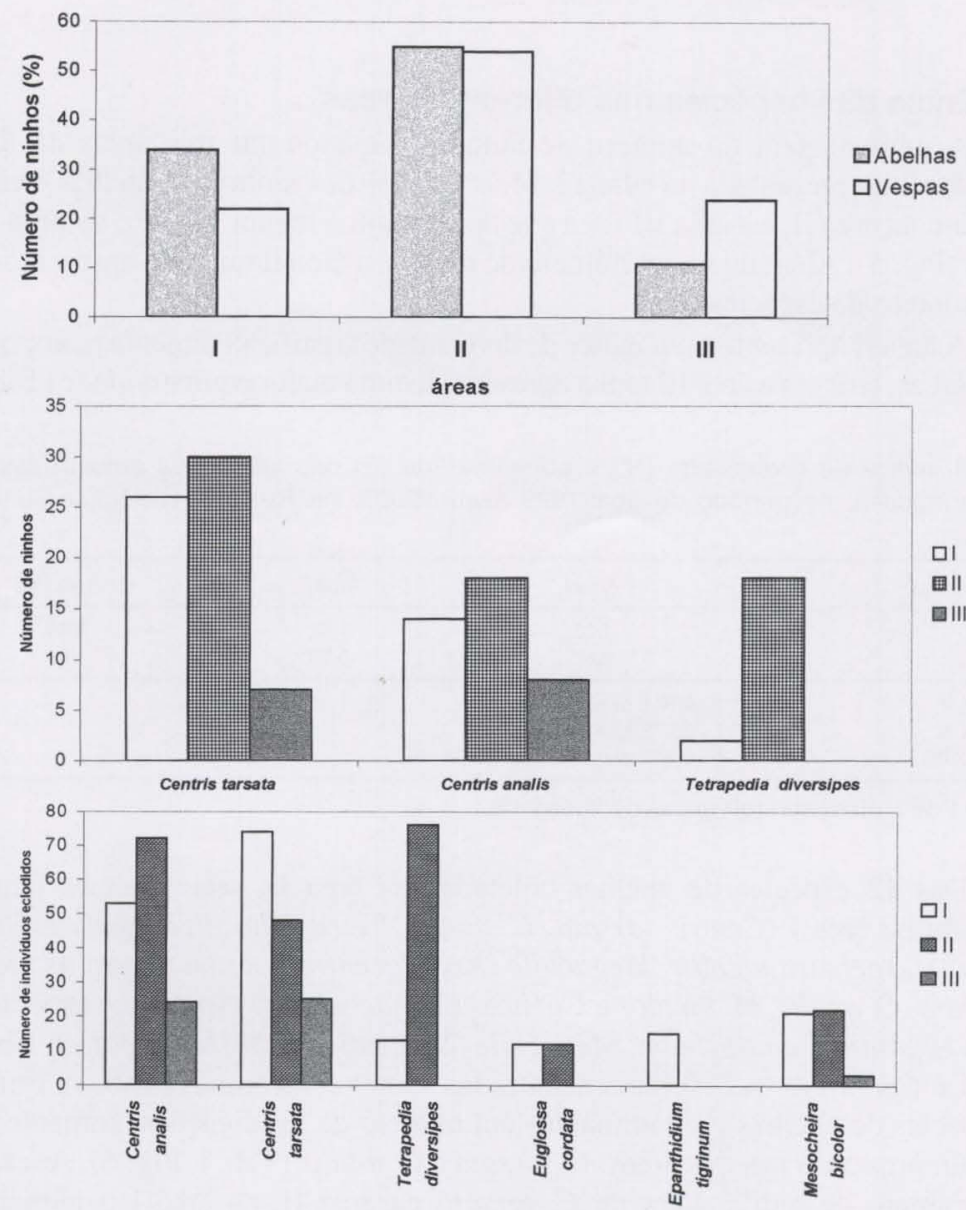

Figs 5-7. (5) Porcentagem do número de ninhos de abelhas e vespas coletados; (6) número de ninhos, por área, das espécies de abelhas mais abundantes; (7) número de indivíduos, por área, das espécies de abelhas mais abundantes que emergiram dos ninhos coletados durante o período de abril/1999 a abril/2000, na Reserva Biológica Guaribas, Paraíba.

Coelioxys (Neocoelioxys) sp. e Coelioxys sp. 2 parasitaram 10\% dos ninhos de Centris analis. Do total de indivíduos emergidos nos ninhos de Centris analis $2,0 \%$ eram de Coelioxys sp. 2 e 1,3\% de Coelioxys (Neocoelioxys) sp. Nos ninhos de Tetrapedia diversipes foi verificada a presença de uma única espécie parasita do gênero Ephestia Guenée, 1845 (Lepidoptera; Pyralidae; Phycitinae). Entre 26 células de seis ninhos de Euglossa cordata, duas foram parasitadas por uma espécie de Anthrax Scopoli, 1763 (Diptera, Bombyliidae).

\section{Razão Sexual}

Foi observada a seguinte razão sexual nas espécies de abelhas: Centris tarsata (57F, 90M; 1:1,5), Centris analis (72F, 77M; 1:1,06), Tetrapedia diversipes 
(44F, 45M; 1:1,02), Euglossa cordata (5F, 19M; 1:3,8), Megachile (Neoemegachile) brethesi (2F), Megachile (Pseudocentron) sp. (8F), Megachile (Sayapis) dentipes $(2 \mathrm{~F})$, Epanthidium tigrinum $(9 \mathrm{~F}, 6 \mathrm{M} ; 1,5: 1)$, Mesocheira bicolor $(30 \mathrm{~F}, 16 \mathrm{M}$; 1,8:1), Coelioxys (Neocoelioxys) sp. (3M), Coelioxys sp.1 (1M), e Coelioxys sp.2 $(1 \mathrm{~F}, 6 \mathrm{M}, 1: 6)$. Entre as quatro espécies predominantes, somente a razão sexual de C. tarsata foi significativamente diferente de $1: 1\left(X^{2}=7,408 \mathrm{p}<0,01\right)$.

\section{DISCUSSÃO}

No conjunto das três áreas amostradas, foi verificado um maior número de ninhos fundados por abelhas do que por vespas. CAMILLO et al. (1995) também encontraram em três áreas de cerrado uma maior ocorrência de ninhos de abelhas do que de vespas. Porém, os estudos realizados em áreas de floresta de terra firme na Amazônia Central (MORATO \& CAMPOS 2000) e em áreas cultivadas na Alemanha (TSCHARNTKE et al. 1998) apresentaram maior ocupação por vespas do que por abelhas.

O número de espécies de abelhas nidificantes na Rebio Guaribas (12 espécies) foi ligeiramente menor do que aquele encontrado por MORATO \& CAMPOS (2000) em áreas de floresta de terra firme da Amazônia Central (14 spp.), bastante inferior ao número de espécies encontradas por CAMILLO et al. (1995) em áreas de cerrado do sudeste do Brasil (32 spp.), e maior que o encontrado por VIANA et al. (2001) em dunas litorâneas do nordeste do Brasil (7 spp.).

Apesar da relativa padronização do método dos ninhos-armadilha, variações da metodologia dos ninhos-armadilha entre os estudos já realizados como tipo de madeira do ninho armadilha, utilização ou não de tubo de cartolina e número de ninhos-armadilha em cada unidade amostral podem resultar em diferenças na diversidade encontrada de espécies de abelhas nidificantes em cavidades preexistentes, além das diferenças devidas à própria composição e abundância das espécies de cada ambiente estudado.

Entre as 32 espécies de abelhas coletadas por CAmillo et al. (1995), ocorreram cinco espécies em comum com este trabalho: Centris tarsata, Centris analis, Tetrapedia diversipes, Euglossa cordata e Epanthidium tigrinum. Além disso, Centris analis e Tetrapedia diversipes estiveram entre as três espécies mais abundantes no estudo de CAMILLO et al. (1995) e também no presente trabalho. Por outro lado, somente Centris analis também ocorreu nas amostras de MORATO \& CAMPos (2000) em floresta de terra firme da Amazônia Central; e somente duas espécies, Euglossa cordata e Centris tarsata, ocorreram em comum com o estudo de VIANA et al. (2001).

Quanto à variação sazonal das nidificações das espécies de abelhas, a maior abundância de nidificações por Centris tarsata na estação seca (entre agosto a novembro) foi semelhante ao observado por MORATO et al. (1999) na Amazônia. Por outro lado, $C$. analis e $T$. diversipes não apresentaram um padrão definido quanto ao período de maior atividade de nidificação. Todavia, a tendência destas espécies de nidificarem no período chuvoso aproxima-se dos dados de CAMILLO et al. (1995) e PEREIRA et al. (1999) que observaram maior atividade das abelhas e vespas na estação quente e chuvosa. Entretanto, nesses estudos, realizados no 
Sudeste do Brasil, a diminuição da atividade reprodutiva na estação fria e seca, deve-se principalmente às baixas temperaturas nesta estação, o que não é esperado para a Rebio Guaribas pois nesta há uma pequena variação da temperatura ao longo do ano.

Quanto à utilização de diferentes diâmetros dos ninhos-armadilha, MORATO et al. (1999) observaram que $C$. analis utilizou dois diâmetros diferentes de ninhos-armadilha, $0,48 \mathrm{~cm}$ e $0,95 \mathrm{~cm}$, com preferência para os ninhos com diâmetro de $0,48 \mathrm{~cm}$. Por outro lado, FRANKIE et al. (1998) observaram nidificação em diâmetros de 0,65 e 0,8 cm. Na Rebio Guaribas, Centris analis apresentou uma maior preferência $(87,5 \%)$ pelos ninhos com $0,6 \mathrm{~cm}$. CAMILLO (2000) estudando a biologia de Tetrapedia curvitarsis Friese, 1899 observou que esta utilizou os diâmetros $0,6 \mathrm{~cm}$ e $0,8 \mathrm{~cm}$, sendo que mais de $60 \%$ dos ninhos foram no diâmetro de $0,8 \mathrm{~cm}$. Na Rebio Guaribas, $70 \%$ dos ninhos de Tetrapedia diversipes foram observados no diâmetro de $0,6 \mathrm{~cm}$.

Provavelmente as espécies de abelhas, com exceção de Euglossa cordata, preferem tubos com diâmetros onde há um melhor ajuste do seu corpo e da sua célula, de modo que orifícios muito maiores envolvem um maior gasto energético para acomodar a célula e preencher os espaços excedentes com material. Na Rebio Guaribas, foi observado que ninhos de C. tarsata apresentavam a parede das células mais espessas, quando construídos em tubos com diâmetros maiores. Conforme COVILLE et al. (1983), o arranjo das células em ninhos de Centris (Heterocentris) Cockerell, 1899 e C. (Xanthemisia) Moure, 1945, está relacionado com a limitação espacial das cavidades.

Em relação às áreas amostradas, a menor diversidade e abundância de abelhas nidificantes nos ninhos-armadilha na área III (área de mata), pode ter diferentes explicações. Inicialmente, pode-se inferir que a área III realmente apresente uma menor diversidade e abundância de abelhas, apresentando estas uma preferência por áreas abertas como as áreas I e II. FRANKIE et al. (1988), na Costa Rica, relatam que dentre as cinco espécies de Centris nidificantes nos ninhos-armadilha somente $C$. analis não apresentou uma preferência entre formações abertas e áreas de mata, sendo que as demais nidificaram preferencialmente em áreas de mata sombreada, provavelmente devido às temperaturas mais amenas nestes ambientes.

O maior número de nidificações ocorridas pelas espécies de abelhas e vespas na área II pode ser devido à preferência destas por ambientes antropogênicos, como observado por CAMILLO et al. (1995). O local na área II onde foram instalados os ninhos-armadilha consistia de uma antiga cocheira coberta, enquanto os locais de instalação dos ninhos-armadilha na áreas I e III foram sob a sombra de árvores. CAMILlO et al. (1995), em duas áreas antropogênicas e uma área de Cerrado, encontraram uma maior abundância de $C$. analis e Tetrapedia diversipes nas áreas antropogênicas, e $C$. tarsata não apresentou preferência de nidificação por nenhuma das áreas. Na Rebio Guaribas $C$. analis e $C$. tarsata também ocorreram nas três áreas estudadas, porém com uma maior ocorrência nas área II, e Tetrapedia diversipes não ocorreu na área florestada. MORATO \& CAMPOS (2000) também observaram maior abundância de $C$. analis em áreas abertas. 
Outra variável que pode explicar a maior abundância de abelhas e vespas nas áreas I e II, em relação à área III, refere-se ao estrato vegetal das áreas. MORATO et al. (1999) encontraram uma maior freqüência de nidificações (77\%), de quatro espécies de Centris, no estrato superior, entre 8 e 15 metros de altura, do que no estrato inferior, na floresta de terra firme da Amazônia Central. Na Rebio Guaribas, os ninhos-armadilha foram instalados a no máximo dois metros de altura, provavelmente estavam situados nos locais onde existe maior atividade da comunidade de abelhas e vespas nas áreas I e II, enquanto que na área III o estrato preferencial deve estar situado mais próximo ao dossel.

GATHMANN et al. (1994) sugerem que a maior diversidade de abelhas encontradas em uma determinada área está relacionada com a diversidade e abundância de recursos florais nesta área. Como a área II possui elementos comuns às áreas de savana e mata fechada, esta apresenta uma maior diversidade floral, o que pode ter propiciado também uma maior diversidade e abundância de abelhas nos ninhos-armadilha.

Mesocheira bicolor foi a espécie parasita mais abundante, com um efeito negativo importante sobre a população de Centris tarsata, considerando que mais de $50 \%$ dos ninhos de $C$. tarsata foram parasitados e $23,2 \%$ dos indivíduos emergidos nesses ninhos pertenciam à esta espécie. VINSON et al. (1987) também observaram uma alta taxa de parasitismo (59\%) por Mesoplia Lepeletier, 1841 nos ninhos agregados de Centris (Centris) flavofasciata Friese, 1900. A presença de vários ninhos-armadilhas próximos provavelmente possibilitou a alta taxa de parasitismo por Mesocheira bicolor. WCISLO \& CANE (1996) sugerem que abelhas solitárias com ninhos próximos facilita a ação dos parasitas.

VINSON \& FRANKIE (1991) especulam que um aumento da atividade de parasitas, como Mesoplia, nos ninhos de Centris (Centris) aethyctera Snelling, 1974, além de diminuir o número de células produzidas, pode aumentar o abandono dos ninhos pela espécie fundadora. O baixo valor da média do número de indivíduos emergidos de C. tarsata, principalmente na área II, também poderia estar relacionado a este fenômeno.

A maior abundância de Mesocheira bicolor foi durante a estação seca correlacionado com a maior taxa de nidificação de Centris tarsata. É interessante notar que não foi verificado nenhum caso de Mesocheira bicolor nos ninhos de Centris analis, apesar desta espécie parasita ter sido mencionada como um parasita abundante nos ninhos de $C$. analis no cerrado do estado de São Paulo por JESUS \& GARÓFALO (2000).

Centris analis e Tetrapedia diversipes apresentaram uma razão sexual próxima de 1:1 do número de machos e fêmeas emergidos nos ninhos. MORATO et al. (1999) também observaram em três espécies de Centris, incluindo C. analis, e CAMILlo (2000) em Tetrapedia curvitarsis, uma razão sexual próxima de 1:1.

Entre as quatro espécies mais abundantes, apenas $C$. tarsata não apresentou uma proporção sexual eqüitativa. A proporção sexual de 1:1,5 de Centris tarsata e 1: 1,8 de Mesocheira bicolor pode estar relacionado ao alto grau de parasitismo da última espécie. Ainda que os ninhos de Centris tarsata não parasitados também apresentaram proporção entre machos e fêmeas semelhante. PEREIRA et al. (1999) 
também observaram, em uma de suas áreas de estudo, uma razão sexual desequilibrada dos indivíduos emergidos nos ninhos de $C$. (Hemisiella) vittata (Lepeletier, 1841). Não foi possível inferir algo sobre as demais espécies devido ao pequeno número de ninhos e indivíduos coletados.

GARÓFALO (2000) comenta sobre a importância da utilização dos dois métodos de amostragem de abelhas, ninhos-armadilha e coletas com rede entomológica sobre as flores, uma vez que os dados obtidos por um dos métodos podem complementar os do outro.

Para uma maior eficiência no estudo das abelhas nidificantes em cavidades preexistentes, utilizando os ninhos-armadilha, algumas variações no método poderiam proporcionar a coleta de uma maior diversidade e abundância de espécies.

Maior número de réplicas das unidades amostrais, distribuídas em diferentes regiões do local de estudo, amostragem em diversos estratos, ninhos-armadilha confeccionados com diferentes materiais (e.g. bambus e blocos de madeira de diversos diâmetros) e períodos mais longos de amostragem, podem alterar o número de espécies nidificantes e as taxas de nidificação.

AGRADECIMENTOS. Agradecemos aos Drs Fernando Silveira, Fernando C.V. Zanella, Danúncia Urban, Márcio Luís de Oliveira e Pe. J.S. Moure, pela identificação das abelhas. À Dra Isabel Alves-dos-Santos, Dr. Clemens Schlindwein e ao Prof. Dr. Fernando C.V. Zanella pelo apoio e sugestões. À CAPES pela bolsa concedida ao primeiro autor. Ao Ibama pela licença de coleta e ao pessoal da Reserva Biológica Guaribas (REBIO) pelo apoio logístico, particularmente ao administrador, biólogo Marcelo Marcelino.

\section{REFERÊNCIAS BIBLIOGRÁFICAS}

Beyer, W.N.; G.W. Miller \& W.J. Fleming. 1987. Populations of trap-nesting wasps near a major source of fluoride emissions in Western Tennessee. Proc. Entomol. Soc. Wash. 89 (3): 478-482.

Camillo, E.; C.A. Garófalo; J.C. Serrano \& G. Muccilo. 1995. Diversidade e abundância sazonal de abelhas e vespas solitárias em ninhos armadilhas (Hymenoptera, Apocrita, Aculeata). Revta bras. Ent. 39 (2): 459-470.

CAmillo, E. 2000. Biologia de Tetrapedia curvitarsis em ninhos armadilha (Hymenoptera: Apidae: Tetrapediini). An. Encontro Abelhas, Ribeirão Preto, 4, p. 103-110.

Coville, R.E.; G.W. Frankie \& S.B. Vinson. 1983. Nests of Centris segregata (Hymenoptera: Anthophoridae) with a review of the nesting habitats of the genus. Jour. Kans. Entomol. Soc. 56: 109-122.

FrANKIE, G.W.; S.B. VinSON; L.E. NeWSTRON \& J.F. BARTHELL. 1988. Nest site and habitats preferences of Centris bees in the Costa Rican dry forest. Biotropica 20 (4): 301-310.

FRANKIE, G.W.; L.E. NEWSTROM; S.B. Vinson \& J.F. BARTHELL. 1993. Nesting-habitat preferences of selected Centris bee species in Costa Rican Dry Forest. Biotropica 25 (3): 322-333.

Frankie, G. W.; R.W. ThorP; M. Newstrom-Loyd; M.A. RizZARD; J.F. BARTHEL; T.L. Griswold; K. JONG-YOON \& S. KAPPAGODA. 1998. Monitoring solitary bees in modified wildland habitats: implications for bee ecology and conservation. Environ. Entomol. 27 (5): 1137-1148.

Garófalo, C.A. 2000. Comunidades de abelhas (Hymenoptera, Apoidea) que utilizam ninhos-armadilhas em fragmentos de matas do Cerrado de São Paulo. An. Encontro Abelhas, Ribeirão Preto, 4, p. 121-128.

Garófalo, C.A.; E. Camillo \& J.C. Serrano. 1989. Espécies de abelhas do gênero Centris (Hymenoptera, Anthophoridae) nidificando em ninhos armadilhas. Ci. Cult. 41: 799.

Revta bras. Zool. 19 (Supl. 1): 101 - 116, 2002 
Garófalo, C.A.; E. Camillo; J.C. Serrano \& J.M.M. Rebêlo. 1993. Utilization of trap nests by Euglossini species. Rev. Brasil. Biol. 53: 177-187.

Gathmann, A.; H.J. Greiler \& T. TsCharnTKE. 1994. Trap-nesting bees and wasps colonizing set-aside fields: sucession and body size, management by cutting and sowing. Oecologia 98: 8-14.

HUTCHESON, K. 1970. A test of comparing diversities based on the Shannon formula. Jour. Theor. Biol. 29: 151-154.

Jesus, B.M. \& C.A. GaRófalo. 2000. Nesting behaviour of Centris (Heterocentris) analis (Fabricius) in southeastern Brazil (Hymenoptera, Apidae, Centridini). Apidologie 31 (4): 503-515.

KrombeIN, K.V. 1967. Trap-nesting wasps and bees: life history, nests, and associates. Washington, DC, Smithsonian Institution Press, VI+570p.

LUDWIG, J.A. \& J.F. REYNOLDS. 1988. Statistical ecology: a primer on methods and computing. New York, John Wiley \& Sons Inc., 338p.

MAGURRAN, A.E. 1988. Ecological diversity and its measurement. Princenton, Princeton Univ. Press, $179 \mathrm{p}$.

Michener, C.D. 2000. The Bees of the World. Baltimore, The Johns Hopkins University Press. 913p.

MMA (MINISTÉRIO DO MEIO AMBIENTE). 2000. Avaliação e ações prioritárias para a conservação da biodiversidade da Mata Atlântica e Campos Sulinos. Brasília, Ministério do Meio Ambiente e outros, 40p.

Morato, F.E.; M.C. GarCia \& L.A. DE O. CAmpos. 1999. Biologia de Centris Fabricius (Hymenoptera, Anthophoridae, Centridini) em matas contínuas e fragmentos na Amazônia Central. Revta bras. Zool. 16 (4): 1213-1222.

Morato, F.E. \& L.A. DE O. CAMPos. 2000. Efeitos da fragmentação florestal sobre vespas e abelhas solitárias em uma área da Amazônia Central. Revta bras. Zool. 17 (2): 429-444.

NIMER, E. 1979. Climatologia do Brasil. Rio de Janeiro, IBGE, 421p.

Oliveira-Filho, A.T. \& D.A. CaRvalho. 1993. Florística da vegetação do extremo Norte do litoral da Paraíba. Rev. Brasil. Bot. 16 (1): 115-130.

Pereira, M.; C.A. Garófalo; E. Camillo \& J.C. Serrano. 1999. Nesting biology of Centris (Hemisiella) vittata Lepeletier in southern Brazil (Hymenoptera, Apidae, Centridini). Apidologie 30: $327-338$.

RouBIK, D.W. 1989. Ecology and natural history of tropical bees. Cambridge, Cambridge Univ. Press, $\mathrm{X}+514 \mathrm{p}$.

Roig-Alsina, A. \& C.D. Michener. 1993. Studies of the phylogeny and classification of long-tongued bees (Hymenoptera: Apoidea). Univ. Kansas Sci. Bull. 55 (4): 123-173.

Sakagami, S.F., Laroca, S. \& J. S. Moure. 1967. Wild bee biocenotics in São José dos Pinhais (PR), South Brazil. Preliminary report. Jour. Fac. Sci. Hokkaido Univ. Ser. VI, Zoology 16 (2): 253-291.

SALGAdo, O.A.; S.J. Filho \& L.M.C. GonçALVES. 1981. As regiões fitoecológicas, sua natureza e seus recursos econômicos. Estudo fitogeográfico, p. 485-544. In: Projeto RADAMBRASIL. Levantamento de Recursos Naturais. Rio de Janeiro, IBGE, Vol. 23, Folhas SB 24/25, 744p.

SERRANo, J.C. \& C.A. Garófalo. 1978. Utilização de ninhos artificiais para o estudo bionômico de abelhas e vespas solitárias. Ci. Cult. 30: 597.

TSCharntKe, T.; A. Gathmann \& I. STEFFandewenter. 1998. Bioindication using trap-nesting bees and wasps and their natural enemies: community structure and interactions. Jour. Appl. Ecol. 35: 708-719.

VIANA, B.F.; F.O. Silva \& A.M.P. KLEINERT. 2001. Diversidade e sazonalidade de abelhas solitárias (Hymenoptera: Apoidea) em dunas litorâneas no nordeste do Brasil. Neotrop. Entomol. 30 (2): 245-251.

Vinson, S.B. \& G.W FrankIE. 1991. Nest variability in Centris aethycera (Hymenoptera: Anthophoridae) in response to nesting site conditions. Jour. Kans. Entomol. Soc. 64 (2): 156-162.

Vinson, S.B.; G.W. Frankie \& J. BARThell. 1993. Threats to the diversity of solitary bees in a Neotropical Dry forest in Central America, p. 53-81. In: J. LASAlle \& I.D. GAuld (Ed.). Hymenoptera and Biodiversity. Wallingford, $C A B$ International, $X X+348 p$. 
Vinson S.B.; G.W. Frankie \& R.E Coville. 1987. Nesting habitats of Centris flavofasciata (Hymenoptera: Apoidea: Anthophoridae) in Costa Rica. Jour. Kansas Entomol. Soc. 60: 249-263. WCISLO, W.T. \& J.H. CANE. 1996. Floral resorce utlization by solitary bees (Hymenoptera: Apoidea) and explotation of their stored foods by natural enemies. Annu. Rev. Entomol. 41: 257-286.

WiLlians, I.H. 1996. Aspects of bee diversity and crop pollination in the European Union, p. 63-80. In: A. Matheson; S.L. Buchmann; C. O’Toole; P. Westrich \& I.H. Willians (Eds). The Conservation of Bees. London, Academic Press, XI+254p.

Recebido em 19.X.2001; aceito em 03.VI.2002. 